\author{
R. AGARWAL ${ }^{1, \infty}$ \\ C.M. LIEBER ${ }^{2}$
}

\section{Semiconductor nanowires: optics and optoelectronics}

\author{
${ }^{1}$ Department of Materials Science and Engineering, University of Pennsylvania, Philadelphia, \\ PA 19104-6272, USA \\ 2 Department of Chemistry and Chemical Biology, and Division of Engineering Applied Sciences, \\ Harvard University, Cambridge, MA 02138, USA
}

\author{
Received: 20 June 2006/Accepted: 14 July 2006 \\ Published online: 30 September 2006 • C Springer-Verlag 2006
}

ABSTRACT Single crystalline semiconductor nanowires are being extensively investigated due to their unique electronic and optical properties and their potential use in novel electronic and photonic devices. The unique properties of nanowires arise owing to their anisotropic geometry, large surface to volume ratio, and carrier and photon confinement in two dimensions (1D system). Currently, tremendous efforts are being devoted to rational synthesis of nanowire structures with control over their composition, structure, dopant concentration, characterization, fundamental properties, and assembly into functional devices. In this article we will review the progress made in the area of nanowire optics and optoelectronic devices, including diodes, lasers, detectors, and waveguides, and will outline the general challenges that must be overcome and some potential solutions in order to continue the exponential progress in this exciting area of research.

PACS 78.67.-n; 78.60.Fi; 78.55.-m; 42.55.-f; 78.67.Lt

\section{$1 \quad$ Introduction}

The shrinking of devices to smaller length scales in the microelectronics industry is becoming prohibitively expensive due to the physical limits imposed by standard lithography techniques [1]. As miniaturization of organized objects and devices remains the cornerstone for creating systems with enhanced functionality (greater computational power and speed, etc.), it is becoming increasingly clear that non-traditional approaches for creating and assembling devices will provide the keys to unlocking the limitless potential of next-generation computing, as well as new opportunities across scientific disciplines $[2,3]$. Chemists traditionally have been working on arranging and manipulating matter at the atomic scale with exquisite control over the spatial arrangement of atoms to form novel molecules. Utilizing such a "bottom up" paradigm to create and assemble devices from a specific arrangement of atoms or molecules has the potential to overcome the limitations of the conventional "top down" lithographic approaches by providing a methodology for control over lattice compositions, scaling of devices to the molecular scale, and minimal surface roughness. In particular,

Fax: +1 215573 2128, E-mail: riteshag@seas.upenn.edu semiconductor nanowires (NWs) and nanotubes (NTs) offer a unique approach for the bottom-up assembly of electronic and photonic devices with the potential for on-chip integration of non-silicon based photonics with silicon nanoelectronics [4-9]. The unique geometries of NWs and NTs enable them to function as both active device elements and interconnects, which can lead to highly integrated device structures. However, the inability to control the electronic properties of NTs during synthesis and the difficulties associated with manipulating individual NTs presents a significant challenge in developing NT-based integrated devices.

By contrast, the ability to rationally synthesize NWs with precisely controlled and tunable chemical composition, size, structure and morphology and to accurately dope them with both $p$ and $n$ type dopants has opened up opportunities for assembling almost any kind of functional nanosystem ranging from integrated solid-state photonics and electronics to biological sensors [10-17]. For example, NWs have been organized into field effect transistors [18], bipolar junction transistors [19], integrated logic calculators [20], high frequency ring oscillators [21], and even sensitive biological and chemical sensors [22], with detection limits down to single virus sensitivity [23]!

Nanowire optics is a particularly exciting frontier due to the ability to precisely control nanowire composition and hence the resulting bandgap, which cannot be accomplished with nanotubes. NWs synthesized from direct bandgap semiconductors have shown tremendous promise for assembling subwavelength nanophotonic devices for the generation, waveguiding, and detection of light at the nanoscale [24]. Nanophotonic devices such as light emitting diodes (LEDs) [25], waveguides [26, 27], electrically driven single NW lasers [28], photodetectors [29, 30], and avalanche photodiodes (APDs)[31], have all been successfully demonstrated. NW-based photonic systems in addition to miniaturization of devices provide many interesting and novel device concepts in comparison to planar technology. The potential for co-assembling nanoscale light sources and detectors, fabricated from a variety of different materials, opens up unique opportunities for integrated photonic systems as well as the integration of nanophotonic systems with silicon micro- and nano-electronics. In this article, we will review the significant progress made in the field of semiconductor NW optics and photonic devices and discuss the scientific and technical challenges that lie ahead. 
2

\section{Nanowire synthesis, characterization and assembly}

Rational synthesis of nanostructures with precise control over geometric parameters and chemical compositions is the most fundamental factor for creating, understanding, and developing the bottom up paradigm for future technologies. NWs are typically synthesized by the vapor-liquidsolid growth mechanism, in which a metal nanoparticle functions as a catalyst for one-dimensional wire growth $[10,11$, 32]. The gas phase reactants mix with the catalyst to form a supersaturated melt, at which point the reactants precipitate via nucleation, and subsequent axial elongation of a crystalline NW occurs. Single crystalline NWs can be synthesized with excellent control over geometric parameters, chemical composition, and dopant concentration from practically any semiconducting material including complex ternary and quaternary materials with diameters ranging from few nanometers to $200 \mathrm{~nm}$ and lengths ranging from few micrometers to $100 \mu \mathrm{m}$ with typical aspect ratios of $10^{3}[6,7]$.

One of the grand challenges of nanoscience is directed assembly of nanoscale components into functional, large scale hierarchical architectures. Integration of nanoscale components into useful devices is non-trivial and requires placing them into specific locations with desired configurations. Current efforts in assembly of nanostructures have focused on techniques such as flow alignment using microfluidic channels [33], lithographically patterned substrates [34], Langmuir-Blodgett films [35], magnetic [36] and electric field assisted alignment [37]. These techniques have the potential for assembling nanoscale components into large scale regular arrays; however they lack the ability to place individual components into precise locations. An ideal assembly technique would be one that enables precise spatial positioning of individual components with the potential for large scale parallel processing. Recently progress has been made toward this goal by assembling NWs in solution with arrays of holographic optical traps (HOTs) [38]. The HOT technique can create hundreds of individually controlled optical traps with the ability to manipulate objects in three dimensions in real time. It has been recently demonstrated that multiple NWs can be trapped simultaneously with control over individual NWs such that they can be isolated, translated, rotated, fused, cut and sequentially deposited onto substrates with HOT arrays. The exciting opportunities provided by the HOT technique in combination with other physical or chemical based assembly techniques will be crucial for creating large-scale integrated yet precisely constructed functional nanosystems in the future.

\section{Optical properties of nanowires}

Photoluminescence (PL) data collected from individual direct-bandgap NWs can reveal a wealth of information, both in terms of characterization of the material and optical properties such as band-edge emission, trap states, radiative efficiency, carrier and photon confinement $[24,28$, 39-49]. A large number of manuscripts have been published reporting the optical properties of NWs and the emerging picture is that individual growth methods and conditions severely affect NW optical properties in terms of emission intensity, spectral width and peak, and the presence of long wavelength components from impurity states that emit radiatively. A variety of NWs made from materials including $\mathrm{ZnO}$ [40], $\mathrm{ZnS}$ [41], GaN [42], CdS [28,46], CdSe [43], ZnSe [44], InP [45], and GaAs [46], have been extensively studied by steady state and transient PL spectroscopy. High quality NWs synthesized under controlled conditions at high temperatures typically emit brightly with a spectral peak near the band-edge. The width of the PL spectra reveals critical information about the quality of the NWs with narrow widths $(\sim 20-30 \mathrm{~nm})$ corresponding to high optical quality of NWs (most likely due to better surface properties, and fewer shallow impurities). For example as shown in Fig. 1b, PL from high-quality CdS NWs typically has a width of $\sim 25 \mathrm{~nm}[28]$ in comparison to GaAs [46] or InP NWs [45] which may have widths ranging from $50-100 \mathrm{~nm}$. This difference is due to the better surface properties of II-VI materials (Fig. 1a) in general in comparison to III-V materials, and since the NWs have high surface area, it affects its optical properties more dramatically than bulk systems. Similarly, the presence of deep traps in the NW material leads to emission at wavelengths much longer than the band-edge emission, and can be minimized by optimizing growth conditions for almost all NW materials. Low-temperature PL spectroscopy on $\mathrm{CdS}$ [49] and $\mathrm{ZnO}$ NWs [24] reveal strong emission from exciton states, which attests to the high optical quality of NWs grown by relatively inexpensive techniques in comparison to molecular beam expitaxy and other ultra-high vacuum techniques. Transient PL spectroscopy of high quality NWs $[49,50]$ has typically revealed rapid sub-nanosecond excited state decay lifetimes (Fig. 1c), which is also a strong indicator of the presence of fewer trap states which otherwise would cause multi-exponential excited state decay with extremely long (nanoseconds to microsecond) timescales. Needless to say, narrow and spectrally pure PL spectra of NWs are extremely critical for creating efficient photonic devices.

Optical absorption and emission from NWs has been found to be predominantly polarized along the long axis of the wires [29]. The observed polarization anisotropy is a purely classical phenomenon arising from the anisotropic geometry of the NWs - where the diameter of the wire is much smaller than the wavelength of light but the length is much larger - and also due to the large dielectric mismatch between the free standing NW and its surrounding environment. Polarized emission from free standing NWs is a very general phenomenon and has been observed on a variety of NW systems with polarization anisotropy determined largely by the NW dielectric constant than its physical dimensions [50,51]. For InP the polarization ratio was reported to be as high as $90 \%\left(\varepsilon_{\mathrm{InP}}=12.4\right)$ [29] whereas for $\mathrm{CdS}$ the ratio is $\sim 60 \%$ $\left(\varepsilon_{\mathrm{CdS}}=7.2\right)$ [31] and was relatively insensitive to the NW diameter and length (Fig. 1d).

PL from NWs also exhibit quantum confinement phenomena due to the radial confinement of carriers if the diameter of the NW becomes comparable to the exciton Bohr radius of the semiconductor. In InP NWs, a systematic blue shift of PL emission was observed from $900 \mathrm{~nm}(d \sim 60 \mathrm{~nm})$ to $800 \mathrm{~nm}$ $(d \sim 10 \mathrm{~nm})$ and was quantitatively explained by an effective mass model using particle-in-a-cylinder wave functions 

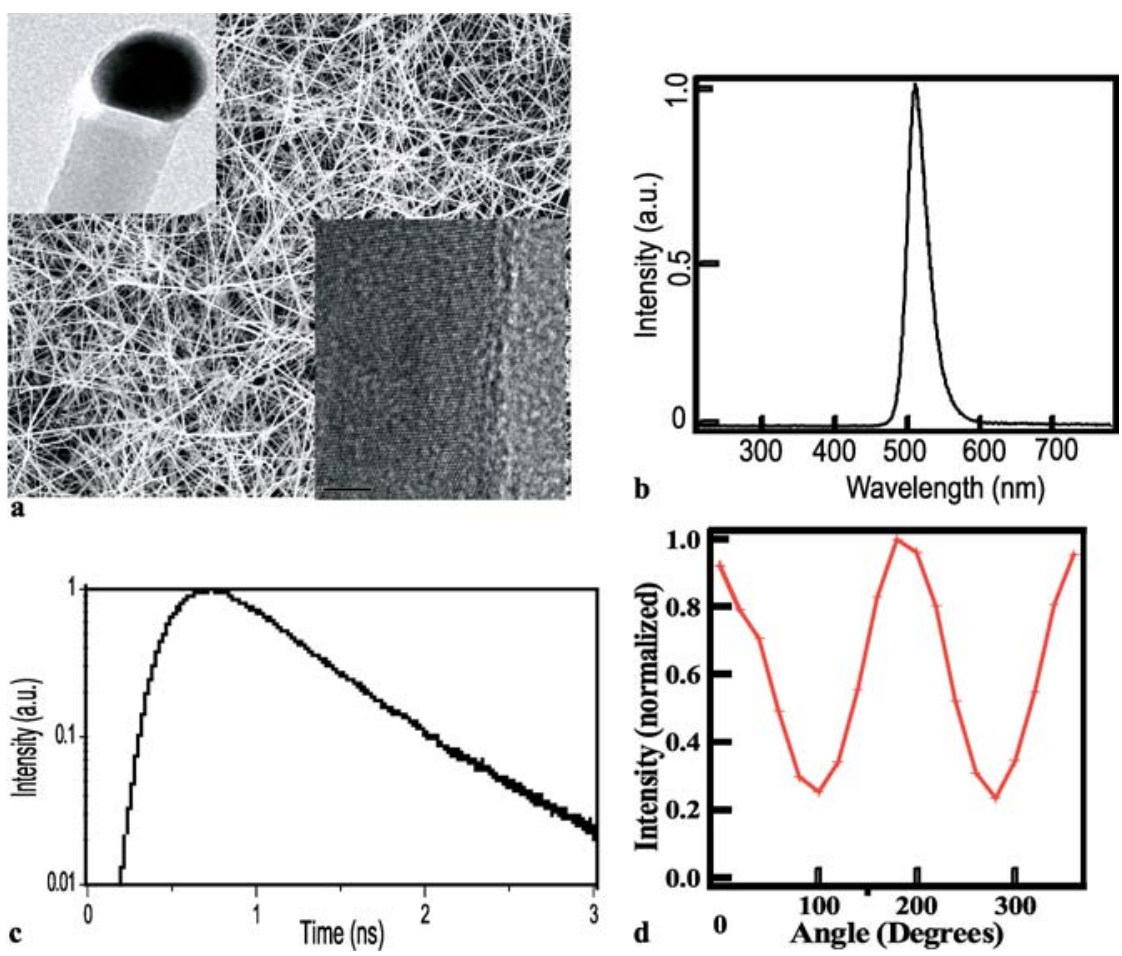

FIGURE 1 (a) SEM image of CdS NWs grown on a $\mathrm{Si}$ substrate. The top inset is a TEM image of a CdS NW with a Au catalyst at the tip. The bottom inset is a high resolution TEM image showing the lattice-resolved image of CdS NW. Scale bar, $10 \mathrm{~nm}$. (b) PL spectrum of a single CdS NW excited with a $266 \mathrm{~nm}$ wavelength laser source. (c) Transient PL decay of a single CdS NW excited with a frequency doubled, 250 fs Ti: sapphire laser pulse. (d) Polarization dependent PL intensity of a single CdS NW with the polarizer placed in front of the CCD detector. The maximum signal is obtained when the polarizer is parallel to the NW long axis while minimum signal is obtained when the polarizer is perpendicular to the NW long axis for carriers $[45,52]$. The prospect of tuning the electronic and optical properties of NWs with size offers tremendous opportunities for fundamental studies of low dimensional systems and assembly of novel devices utilizing quantum properties of the system.

If the diameter of the NW exceeds the wavelength of light that can propagate in a medium $(\sim \lambda / 2 n$, where $\lambda$ is the wavelength of light in vacuum and $n$ is the refractive index of the NW material), then the PL image of NWs uniformly excited by light reveals enhanced emission from the NW ends (Fig. 2a) [28]. If a sharply focused laser is used to excite the NW, then emission is observed only from the laser excitation area and from the NW ends, which shows that NWs can function as efficient optical waveguides (Fig. 2b inset). The large dielectric mismatch between the NW and its surrounding environment causes tight photon confinement in sub-wavelength sized NWs, and light can be waveguided over large distances with very little loss [24,28]. The PL spectrum from the NW body vs. NW ends reveals very interesting differences as shown in Fig. 2b: emission from the NW body was observed to be featureless whereas the NW end showed periodic modulation in emission intensity. The modulated spectrum from NW ends is due to the Fabry-Perot optical cavity modes of the NW because the ends of the NW, which can be perfectly cleaved via a mild sonication process, act as partially reflecting mirrors that provide optical feedback and hence create standing optical waves in the NW cavity. The mode spacing was observed to be inversely proportional to the length of the NW, as expected for a Fabry-Perot cavity. The observation of Fabry-Perot modes in single NW optical cavities suggests that lasing can be sustained in NWs. Indeed, lasing stimulated by optical pumping in epitaxially grown ensemble of ZnO NWs was first reported by P. Yang's group at Berkeley [53], followed by observation of lasing in indi- vidual ZnO NWs [54]. Subsequently there have been many reports of lasing in single NWs from different materials including CdS [28], GaN [55], and ZnS [56]. Figure 2c shows the collapse of a broad PL spectrum to single mode lasing above the lasing threshold under intense optical excitation. Lasing was only observed from NW ends and not from the NW body, which is consistent with the fact that an optical feedback mechanism is necessary for lasing.

Detailed temperature-dependent optically-pumped studies of single CdS NW lasers revealed that a highly efficient exciton-exciton scattering phenomena is responsible for lasing from $4.2 \mathrm{~K}$ until $70 \mathrm{~K}$, whence the lasing mechanism shifts to an exciton-optic phonon scattering mechanism at higher temperatures [49]. Nevertheless, the mechanism of lasing in CdS NWs remained exciton-based at all temperatures, in comparison to the formation of electron-hole plasma, which are created at very high carrier densities due to the screening of excitons that leads to their dissociation into free carriers [57]. Exciton-based lasing in CdS NWs shows that NWs can form efficient cavities to sustain lasing at low threshold powers. This was quantitatively shown by the temperature dependence of the lasing threshold in CdS NWs, and it was observed that the threshold power for NW lasers is $\sim 10$ times less sensitive to temperature than CdS platelets [58]. The less temperature dependence of lasing threshold power and exciton-based lasing is likely due to very strong photon confinement ( $\sim 50 \%$ mode confinement) in NW cavities, which leads to excellent overlap of the optical mode with optically pumped carriers, a desirable feature for any semiconductor laser. These studies also demonstrated that detailed optical studies provides a powerful tool to unravel the various complex mechanisms operative in NWs and will aid in the development of more efficient lasers and other photonic devices. 

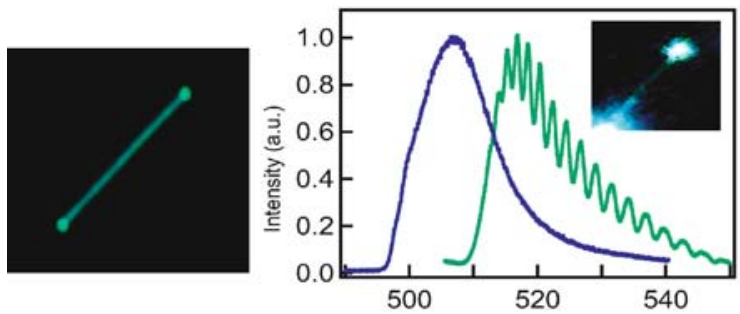

a

b Wavelength $(\mathrm{nm})$

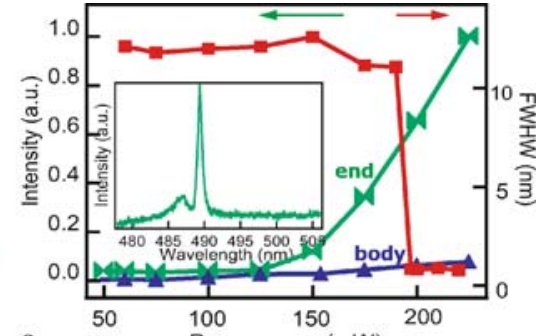

c

Pump power $(\mathrm{mW})$
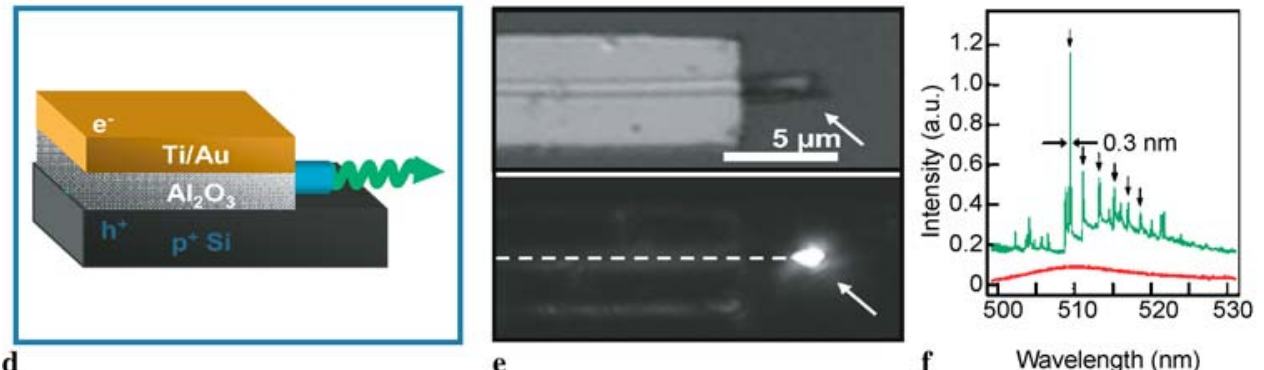

f

Wavelength $(\mathrm{nm})$

FIGURE 2 CdS NW optical and electrically pumped laser. (a) PL image of a single CdS NW showing enhanced emission from the two ends. (b) PL spectra obtained from the CdS NW body (blue) and end (green). (c) Emission intensity and FWHM of emission peaks versus laser pump power. The emission intensity from the nanowire body (blue) is approximately linear in pump power, whereas the emission from the nanowire end (green) exhibits superlinear behavior above the lasing threshold. The FWHM (red) has a nearly constant value of about $12 \mathrm{~nm}$ at low powers, and abruptly narrows to the instrument-resolution value above the lasing threshold. The inset is the lasing spectrum of a CdS NW. (d) Schematic showing the NW electrical injection laser device structure. In this structure, electrons and holes can be injected into the CdS nanowire along the whole length from the top metal layer and the bottom $p$-Si layer, respectively. (e) Top panel shows an optical image of a laser device and the arrow highlights the exposed CdS nanowire end. Scale bar, $5 \mu \mathrm{m}$. Bottom panel shows an electroluminescence image recorded from this device. The dashed line highlights the nanowire position. (f) Electroluminescence spectra obtained from the nanowire end with injection currents of $120 \mu \mathrm{A}$ (red, below lasing threshold) and $210 \mu \mathrm{A}$ (green). The black arrows highlight Fabry-Perot cavity modes with an average spacing of $1.83 \mathrm{~nm}$. The green spectrum is shifted upwards by 0.15 intensity units for clarity. Adapted from [28]

\section{4}

\section{1}

\section{Nanowire optoelectronics}

\section{Electrical generation of light at the nanoscale using subwavelength sources}

Despite the excitement generated from the first demonstration of optically pumped NW lasers, it is desirable that lasing in NWs be achieved by electrical injection for its tremendous technological relevance. To achieve lasing by electrical pumping, efficient injection of carriers in the NW cavity is required. Crossed-NW devices have been previously configured as very efficient $p-n$ junction diodes where rectification of current is clearly observed [19]. If the diode is assembled with a direct bandgap semiconductor NW, then under forward bias light emission is observed from the NW crossing point, where the diode junction is formed and carrier injection and recombination takes place [25]. This concept was first demonstrated for crossed $p-n$ InP NWs and later extended to other materials $[25,59]$. Efficient nanoscale LEDs can also be formed between $p$-Si NWs crossed with NWs made from an $n$-type direct bandgap material [60], but owing to the localized injection of charges and subsequent heat generation at the nanoscale cross-point the devices could not be pumped above lasing threshold. It was clear that uniform injection of carriers along the NW length would be required to achieve electrical pumped lasing.

To enable uniform injection of carriers in a NW optical cavity, $n$-CdS NWs were assembled on heavily doped $p$-Si substrates and contacts to CdS were fabricated by lithographic techniques (the $p$-Si substrate functioned as the second electrode) as shown schematically in Fig. 2d [28]. A thin layer of $\mathrm{Al}_{2} \mathrm{O}_{3}$ was deposited in between the top and bottom contacts to force the current through the $p$-Si $/ n$ - $\mathrm{CdS}$ diode formed at the NW-substrate junction. The device under low forward bias, produced EL with a broad featureless spectrum from the exposed NW end (Fig. 2e, f). Upon increasing the bias, the EL spectrum quickly collapsed into single mode lasing line at $493 \mathrm{~nm}$ with instrument resolution limited line-width of $0.7 \mathrm{~nm}$, clearly demonstrating the possibility of electrically pumped lasing in NW optical cavities. Implicit in this demonstration is the integration of a photonic device with conventional silicon microelectronics, which is otherwise challenging with planar semiconductor technology. Electrically driven lasing in single NWs represents a novel and powerful approach for assembling highly integrated photonic devices with the potential to co-assemble diverse materials such as $\mathrm{GaN}, \mathrm{ZnO}, \mathrm{CdSe}$, and $\mathrm{InP}[60]$ on a common platform to produce lasers and other photonic devices that can cover the entire optical spectrum ranging from the UV to the infrared.

\subsection{Manipulation of light at the nanoscale}

The demonstration of waveguiding in subwavelength scale NWs suggests a promising approach for guiding light at the nanoscale from one part of the chip to another over large distances and in performing key tasks such as communication, computing, and sensing $[26,27]$. Routing of the optical signal can be achieved by coupling subwavelength waveguides directly to the active elements such as LEDs, lasers and detectors. The efficiency of waveguiding in NWs excited by optical excitation was estimated by measuring the loss of output intensity at the other end of 
the long nanowire with efficient waveguiding observed over $0.5 \mathrm{~mm}$ distance $[26,27,61]$. It was noted that the propagation losses were significantly less compared to other waveguiding technologies including plasmonic stuctures [27]. Efficient coupling between waveguides is essential to assemble complex NW optical networks for computing and communication applications. It was observed that the coupling strength of the optical linkages formed when NWs are brought into intimate contact depends on the volume of interaction and the angle of intersection [26, 27]. By using efficient coupling schemes, it has been demonstrated that the lasing emission from NWs can launch pulses of light through other waveguides that are up to a millimeter in length. In addition, waveguiding in NWs in a liquid environment was demonstrated, which may provide a unique approach for optics-based sensing in solution [61].

Efficient coupling of light into subwavelength waveguides has been a challenge for all systems such as plasmonic and photonic crystal guides, and includes techniques such as using fiber tapers or evanescent wave coupling. In NWs, waveguide modes can be excited by electrical injection of carriers in a $p-$ $n$ junction diode configuration (discussed above) [28] and is a powerful approach for using NW waveguides for generation and transport of photons to different regions on a chip with minimal loss. Recently it has also been demonstrated that the intensity of waveguided modes in NWs can be modulated electro-optically [62], and coupling of NW emission with lithographically defined photonic crystal structures leads to localized emission from engineered defects and light suppression in regions of the photonic crystal [63]. However, it will be desirable to incorporate photonic crystal structures along the length of NWs during synthesis, for example, by composition modulation, in order to achieve very high device densities $[13,14]$. In another interesting study of manipulating emission from NWs, quantum dots were assembled inside NWs and single photon emission was observed from the dots [64], which opens up possibilities for researching quantum optics in NWs and obtaining electrically driven single photon sources.

\subsection{Detection of light at the nanoscale using subwavelength detectors}

Detection of light at the nanoscale poses a fundamental challenge owing to the intrinsically small size of the nanostructures, which leads to extremely small photon absorption cross-section. Single NWs and NTs configured as photoconductors are not very sensitive photodetectors due to their small diameter $[29,30,65]$, and increasing their size for enhancing the sensitivity defeats the purpose of developing the technology for highly miniaturized nanodevices. This therefore presents a dilemma for solving the problem of highly sensitive photodetection at the nanoscale without increasing the size of the nanostructure.

Cross-NW devices configured as $p-n$ junction diodes show current rectification as discussed in the previous section. However, detailed analysis of reverse biased crossed NW $p-n$ junctions has received less attention. Under reverse bias, $p-n$ junctions break down via two mechanisms; Zener and avalanche breakdown. Zener breakdown occurs in heav- ily doped semiconductors where the depletion width is small and the carriers tunnel from one side of the junction to the other under high reverse bias [66]. There is no carrier multiplication during Zener breakdown and the photocurrent is typically constant with increasing reverse bias, until junction breakdown occurs. Avalanche breakdown typically occurs in low doped semiconductors where the large depletion width prevents tunneling of carriers across the junction. However, with increasing reverse bias, the carriers accelerate in the presence of an electric field, and can attain very high kinetic energies such that if they collide with the crystal lattice they can knock electrons from their bonds, creating additional electron/hole pairs and thus additional current. These secondary carriers also accelerate in the presence of an electric field, and the process repeats itself creating an avalanche of new carriers producing large current. The photocurrent generated in a reverse biased avalanche diode is therefore strongly dependent on the bias applied, in contrast to a Zener breakdown device.

It seems clear that in order to increase the sensitivity of photon detection at the nanoscale without compromising the small size of the nanodevice, an amplification process has to be engineered in the device to make up for the limitations of small photon absorption cross-section. In a recent demonstration, crossed $p-\mathrm{Si} / n-\mathrm{CdS} \mathrm{NW}$ diodes were assembled and the dopant concentration in $p$-Si NW was kept very low to minimize the depletion width and hence facilitate avalanche multiplication [31]. The device showed current rectification, and under forward bias produced EL from the crossing region characteristic of emission from CdS NW (Fig. 3a). The device under reverse bias showed exceptionally low dark currents $(<100 \mathrm{pA})$ with a sharp breakdown occurring at $\sim-9.0 \mathrm{~V}$. Upon illuminating the device with $488 \mathrm{~nm}$ light, a large reverse-bias dependent photocurrent was observed, which is indicative of avalanche breakdown phenomenon. The multiplication factor, $M$, was estimated to be $\sim 5 \times 10^{4}$ for sub-breakdown biases and was largely reproducible, with values of $M$ as large as $7 \times 10^{4}$ obtained. In contrast, single $n-\mathrm{CdS}$ or $p$-Si NW devices exhibited maximum gain of only $3-5$, which strongly suggests that the high gain observed in NW diodes results from an amplification process associated with the $p-n$ junction and not from photoconductivity from individual NWs.

Spatially resolved photocurrent measurements as shown in Fig. 3b performed on the crossed-wire devices with a diffraction limited laser ( $250 \mathrm{~nm}$ spot size) revealed a single, highly localized photocurrent peak located at the position of the crossed NW $p-n$ junction [31]. No photocurrent was observed at the NW-electrode contacts, which is distinct from previous studies [67] where Schottky barriers formed at the contacts have been shown to dominate the photoresponse. In addition, the photocurrent scanning measurement demonstrated that the spatial resolution of the device was at least $250 \mathrm{~nm}$, thereby demonstrating that the detector has subwavelength spatial resolution capabilities.

The photon detection limits of the nanoAPDs demonstrated a large dynamic range from $\mathrm{pW}$ to $\mathrm{mW}$ with the lowest power that was easily measured was $4 \mathrm{pW}$, corresponding to an estimated detection limit of $\sim 75$ photons (Fig. 3c). This very high sensitivity is due to the large avalanche multipli- 

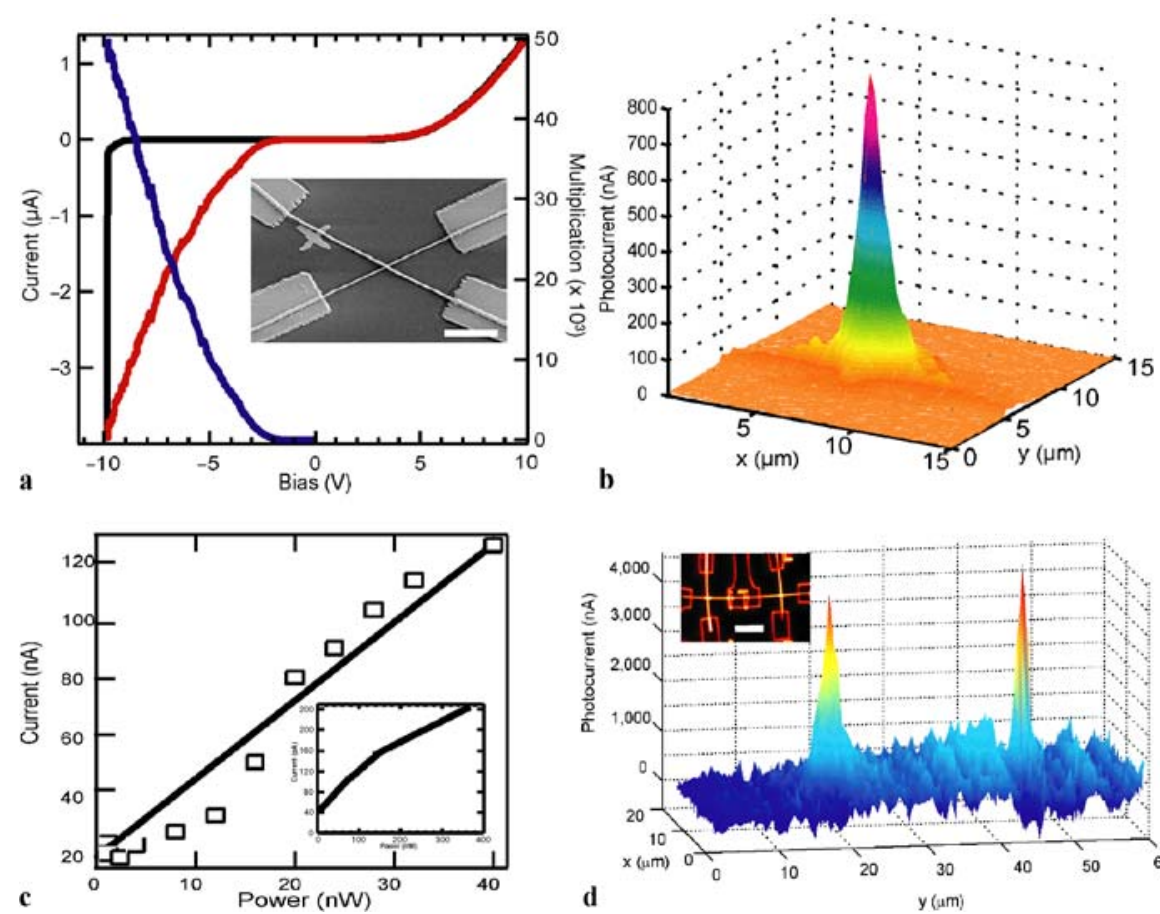

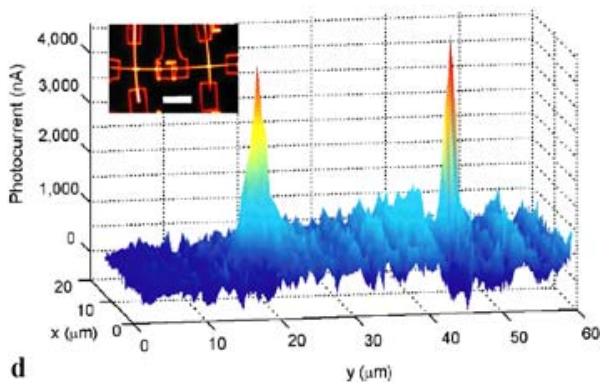

FIGURE 3 Nanowire avalanche photodiode. (a) $I-V$ characteristic of the APD in dark (black line) and illuminated (red line) conditions; the device was illuminated with $500 \mathrm{nW}$ of 488-nm light (red line). The bias-dependent gain (carrier multiplication) is shown by the blue line. The inset shows a scanning electron micrograph of the $n$-CdS/ $p$-Si device; the scale bar is $4 \mu \mathrm{m}$. (b) Plot of the spatially resolved photocurrent from the nanoAPD measured using a diffraction-limited laser; the bias voltage, laser power and scanning step size were $-7 \mathrm{~V}$, $200 \mathrm{nW}$ and $250 \mathrm{~nm}$ (in $x$ and $y$ ), respectively. (c) Detection sensitivity measured at room temperature using a calibrated photon source which was a frequency doubled Ti:sapphire laser at $400 \mathrm{~nm}$ (250-fs pulses; 76- $\mathrm{MHz}$ repetition rate). The inset is the photoresponse in the $0-400 \mathrm{pW}$ power range. (d) Spatially resolved photocurrent measured from the $2 \times 1$ APD array shown in the inset. Both devices were biased at $-10 \mathrm{~V}$ and excited at $488 \mathrm{~nm}\left(200 \mathrm{nW}, \mathrm{Ar}^{+}\right.$-ion laser) with a scanning step size of $1 \mu \mathrm{m}$. Adapted from [31] cation effect, which compensates for the low photon absorption cross-section of nanoscale photodetectors and presents significant improvements over NW photoconductors without carrier multiplication [29, 30, 67].

The temporal response of the nanoAPDs revealed a single photocurrent peak with instrument response limited risetime of $3 \mu \mathrm{s}$, which was much faster than other recently reported NW photodetectors [30,67]. The photoresponse of the devices was also found to be highly sensitive to the polarization of the detected light with polarization ratios of $\sim 60 \%$. This polarization sensitivity arises because $\mathrm{CdS} \mathrm{NW}$ is the predominant absorbing medium and as discussed before, light polarized parallel to long axis of the $\mathrm{CdS}$ is preferentially absorbed. Polarization dependent photoresponse represents a unique feature in comparison to conventional detectors and offers interesting opportunities for creating novel polarization sensitive photonic components at the nanoscale.

Crossed NW device architecture can also be extended to larger arrays with independently addressable devices. This concept was demonstrated by assembling an array consisting of two Si NW s crossing a single CdS NW [31]. Scanning photocurrent data acquired from this array exhibited two localized photocurrent peaks located at the positions of the individual diodes (Fig. 3d), and could be independently controlled with no electrical cross-talk between the devices. The concept can readily be expanded to larger arrays with the possibility of assembling high resolution imaging devices with polarization sensitivity, fast temporal response and extremely high photon sensitivity.

The approach to assemble sensitive crossed NW photodiodes is very general, and flexible, and should be extendable to other semiconductor NW materials, which could tailor sensitivity in all spectral regions [60]. Sensitivity and temporal response of the devices can be further improved by designing $p-i-n$ junctions with better control over the junction electric fields to eventually enable single photon level sensitivity. The features demonstrated for the APDs offer substantial promise in diverse areas ranging from integrated photonics, near-field detection, and high spatial resolution images to medical diagnostics, and sensors.

\section{$5 \quad$ Future outlook}

Impressive progress has been made in the last few years in the area of semiconductor NW growth and characterization of structural, electronic and optical properties, and assembly of devices. Despite the current excitement in the field of NW technology, many challenges remain to be overcome in order to enable unique technologies in the future. Controlled manipulation of matter at the nanoscale should lead to fascinating and novel behavior and should also impact device properties. The ability to fabricate intra-NW heterostructures $[13,14]$ with well defined, atomically abrupt crystalline interfaces should further increase the versatility of NW based electronic and photonic devices and also reduce the load for subsequent assembly processes. Such a synthetic control will be extremely useful for creating precisely defined systems to investigate the effects of quantum confinement on electronic and optical properties of nanostructures resulting from the modification of the electronic density of states, and also aid in developing novel nanophotonic devices. With extensive research in NW synthesis to accurately control dimension and composition, a critical understanding of the modified properties of materials at the nanoscale, and the hierarchical assembly of nanostructures with exquisite spatial control, progress will be made and new and interesting nanosystems with enhanced optical and electronic properties will be assembled with applications ranging from computing, biological/chemical sensing to imaging. 


\section{REFERENCES}

1 International Technology Roadmap for Semiconductors, 2005 report. http://www.itrs.net/Common/2005ITRS/ExecSum2005.pdf

2 R. Compano, L. Molenkamp, D.J. Paul, Technology Roadmap for Nanoelectronics, European Commission IST Program: Future and Emerging Technologies: Microelectronics Advanced Research Initiative; http://nanoworld.org/NanoLibrary/nanoroad.pdf

3 J.R. Heath, P.J. Kuekes, G.S. Snider, R.S. Williams, Science 280, 1716 (1998)

4 C.M. Lieber, Sci. Am. 285, 58 (2001)

5 M. Law, J. Goldberger, P. Yang, Ann. Rev. Mater. Res. 34, 83 (2004)

6 C.M. Lieber, MRS Bull. 28, 486 (2003)

7 Y. Xia, P. Yang, Y. Sun, Y. Wu, B. Mayers, B. Gates, Y. Yin, F. Kim, H. Yan, Adv. Mater. 15, 353 (2003)

8 C. Dekker, Phys. Today 52, 22 (1999)

9 P.G. Collins, P. Avouris, Sci. Am. 283, 62 (2000)

10 A.M. Morales, C.M. Lieber, Science 279, 208 (1998)

11 X. Duan, C.M. Lieber, Adv. Mater. 12, 298 (2000)

12 C. Yang, Z. Zhong, C.M. Lieber, Science 310, 1304 (2005)

13 M.T. Bjork, B.J. Ohlsson, T. Sass, A.I. Persson, C. Thelander, M.H. Magnusson, K. Deppert, L.R. Wallenberg, L. Samuelson, Nano Lett. 2, 87 (2002)

14 L.J. Lauhon, M.S. Gudiksen, D. Wang, C.M. Lieber, Nature 420, 57 (2002)

15 M.S. Gudiksen, J. Wang, C.M. Lieber, J. Phys. Chem. B 105, 4062 (2001)

16 T. Kuykendall, P.J. Pauzauskie, Y. Zhang, J. Goldberger, D. Sirbuly, J. Denlinger, P. Yang, Nature Mater. 3, 524 (2004)

17 J. Goldberger, R. He, Y. Zhang, S. Lee, H. Yan, H. Choi, P. Yang, Nature 422, 599 (2003)

18 Y. Cui, Z. Zhong, D. Wang, W.U. Wang, C.M. Lieber, Nano Lett. 3, 149 (2003)

19 Y. Cui, C.M. Lieber, Science 251, 891 (2001)

20 Y. Huang, X. Duan, Y. Cui, L.J. Lauhon, K. Kim, C.M. Lieber, Science 294, 1313 (2001)

21 R.S. Friedman, M.C. McAlpine, D.S. Ricketts, D. Ham, C.M. Lieber, Nature 434, 1085 (2005)

22 F. Patolsky, C.M. Lieber, Mater. Today 8, 20 (2005)

23 F. Patolsky, G. Zheng, O. Hayden, M. Lakadamyali, X. Zhuang, C.M. Lieber, Proc. Nat. Acad. Sci. USA 101, 14017 (2004)

24 D.J. Sirbuly, M. Law, H. Yan, P. Yang, J. Phys. Chem. B 109, 15190 (2005)

25 X. Duan, Y. Huang, Y. Cui, J. Wang, C.M. Lieber, Nature 409, 66 (2001)

26 M. Law, D.J. Sirbuly, J.C. Johnson, J. Goldberger, R.J. Saykally, P. Yang, Science 305, 1269 (2004)

27 C.J. Barrelet, A.B. Greytak, C.M. Lieber, Nano Lett. 4, 1981 (2004)

28 X. Duan, Y. Huang, R. Agarwal, C.M. Lieber, Nature 421, 241 (2003)

29 J. Wang, M.S. Gudiksen, X. Duan, Y. Cui, C.M. Lieber, Science 293, 1455 (2001)

30 H. Kind, H. Yan, B. Messer, M. Law, P. Yang, Adv. Mater. 14, 158 (2002)

31 O. Hayden, R. Agarwal, C.M. Lieber, Nat. Mater. 5, 352 (2006)

32 R.S. Wagner, W.C. Ellis, Appl. Phys. Lett. 4, 89 (1964)

33 Y. Huang, X. Duan, Q. Wei, C.M. Lieber, Science 291, 630 (2001)

34 B.R. Martin, S.K.St. Angelo, T.E. Mallouk, Adv. Funct. Mater. 12, 759 (2002)

35 D. Whang, S. Jin, Y. Wu, C.M. Lieber, Nano Lett. 3, 1255 (2003)
36 M. Tanase, D.M. Silevitch, A. Hultgren, L.A. Bauer, P.C. Searson, G.J. Meyer, D.H. Reich, J. Appl. Phys. 91, 8549 (2002)

37 L.A. Nagahara, I. Amlani, J. Lewenstein, R.K. Tsui, Appl. Phys. Lett. 80, 3826 (2002)

38 R. Agarwal, K. Ladavac, Y. Roichman, G. Yu, C.M. Lieber, D.G. Grier, Opt. Express 13, 8906 (2005)

39 W.S. Shi, Y.F. Zheng, N. Wang, C.S. Lee, S.T. Lee, Appl. Phys. Lett. 78 $3304(2001)$

40 P. Yang, H. Yan, S. Mao, R. Russo, J. Johnson, R. Saykally, N. Morris, J. Pham, R. He, H.-J. Choi, Adv. Funct. Mater. 12, 323 (2002)

41 Q. Xiong, G. Chen, J.D. Acord, X. Liu, J.J. Zengel, H.R. Gutierrez, J.M. Redwing, L.C.L.Y. Voon, B. Lassen, P.C. Eklund, Nano Lett. 4, 1663 (2004)

42 H.W. Seo, S.Y. Bae, J. Park, H. Yang, K.S. Park, S. Kim, J. Chem. Phys. 116, 9492 (2002)

43 C. Ma, Y. Ding, D. Moore, X. Wang, Z.L. Wang, J. Am. Chem. Soc. 126, 708 (2004)

44 B. Xiang, H.Z. Zhang, G.H. Li, F.H. Yang, F.H. Su, R.M. Wang, J. Xu, G.W. Lu, X.C. Sun, Q. Zhao, D.P. Yu, Appl. Phys. Lett. 82, 3330 (2003)

45 M.S. Gudiksen, J. Wang, C.M. Lieber, J. Phys. Chem. B 106, 4036 (2002)

46 X. Duan, J. Wang, C.M. Lieber, Appl. Phys. Lett. 76, 1116 (2000)

47 S. Bhattacharya, D. Banerjee, K.W. Adu, S. Samui, S. Bhattacharyya, Appl. Phys. Lett. 85, 2008 (2004)

48 N. Panev, A.I. Persson, N. Sköld, L. Samuelson, Appl. Phys. Lett. 83, 2238 (2003)

49 R. Agarwal, C.J. Barrelet, C.M. Lieber, Nano Lett. 5, 917 (2005)

50 M. Bashouti, W. Salalha, M. Brumer, E. Zussman, E. Lifshitz, Chem. Phys. Chem. 7,(1) 102 (2005)

51 J.C. Johnson, H.Q. Yan, P. Yang, R.J. Saykally, J. Phys. Chem. B 107, $8816(2003)$

52 H. Yu, J. Li, R.A. Loomis, L.-W. Wang, W.E. Buhro, Nat. Mater. 2, 517 (2003)

53 M. Huang, S. Mao, H. Feick, H. Yan, Y. Wu, H. Kind, E. Weber, R. Russo, P. Yang, Science 292, 1897 (2001)

54 J.C. Johnson, H. Yan, R.D. Schaller, L.H. Haber, R.J. Saykally, P. Yang, J. Phys. Chem. B. 105, 11387 (2001)

55 J.C. Johnson, H.J. Choi, K.P. Knutsen, R.D. Schaller, P. Yang, R.J. Saykally, Nat. Mater. 1, 106 (2002)

56 J.A. Zapien, Y. Jiang, X.M. Meng, W. Chen, F.C.K. Au, Y. Lifshitz, S.T. Lee, Appl. Phys. Lett. 84, 1189 (2004)

57 T.M. Rice, Solid State Phys. 32, 1 (1977)

58 J.M. Hvam, Phys. Rev. B. 4, 4459 (1971)

59 Z. Zhong, F. Qian, D. Wang, C.M. Lieber, Nano Lett. 3, 343 (2003)

60 Y. Huang, X. Duan, C.M. Lieber, Small 1, 142 (2005)

61 D.J. Sirbuly, M. Law, P. Pauzauskie, H. Yan, A.V. Maslov, K. Knudsen, R.J. Saykally, P. Yang, Proc. Nat. Acad. Sci. USA 102, 7800 (2005)

62 A.B. Greytak, C.J. Barrelet, Y. Li, C.M. Lieber, Appl. Phys. Lett. 87, 151103 (2005)

63 C.J. Barrelet, J. Bao, M. Loncar, H.-G. Park, F. Capasso, C.M. Lieber, Nano Lett. 6, 11 (2006)

64 M.T. Borgstrom, V. Zwiller, E. Muller, A. Imamoglu, Nano Lett. 5, 1439 (2005)

65 J.A. Misewich, R. Martel, Ph. Avouris, J.C. Tsang, S. Heinze, J. Tersoff, Science 300, 783 (2003)

66 B.E.A. Saleh, M.C. Teich, Fundamentals of Photonics (Wiley, New York 1991)

67 Y. Gu, E. Kwak, J.L. Lensch, J.E. Allen, T.W. Odom, L.J. Lauhon, Appl Phys. Lett. 87, 043111 (2005) 